\title{
A trapezoidal wing equivalent to a Janatella leucodesma's wing in terms of aerodynamic performance in the flapping flight of a butterfly model
}

\author{
Kosuke Suzuki \& Masato Yoshino \\ Institute of Engineering, Academic Assembly, Shinshu University, Nagano 380-8553, \\ JAPAN \\ E-mail: kosuzuki@shinshu-u.ac.jp
}

September 2018

\begin{abstract}
Wing planform is one of important factors for lift and thrust generation and enhancement in flapping flight. In our previous study using a simple numerical model of a butterfly, it was found that the wing planform of an actual butterfly (Janatella leucodesma) is more efficient than any of the rectangular or trapezoidal wing planforms. In the present study, we make a hypothesis that the efficient aerodynamic performance of the butterfly's wing can be reproduced by the following four geometrical parameters of wing planform: aspect ratio, taper ratio, position of the rotational axis for the geometric angle of attack, and sweepback angle. In order to test this hypothesis, we explore a trapezoidal wing planform equivalent to the actual butterfly's wing planform in terms of aerodynamic performance in the parameter space consisting of these four parameters. We use a simple butterfly model composed of two rigid thin wings and a rod-shaped body, and calculate the aerodynamic performance of the model by an immersed boundary-lattice Boltzmann method to find such a trapezoidal wing planform. As a result, we find a trapezoidal wing planform which gives almost the same lift, thrust, pitching moment, power, and power-loading coefficients as the actual butterfly's wing planform. Furthermore, in the free flight of the butterfly model with pitching motion control, the flight behavior of the model with the resulting trapezoidal wing planform is almost the same as that with the actual butterfly's wing planform.
\end{abstract}

\section{Introduction}

Butterflies have unique and interesting features compared with other insects. The most conspicuous example is their erratic trajectory and large variation in speed. This behavior suggests that butterflies have outstanding agility and maneuverability, which are attractive features in practical applications such as micro air vehicles (MAVs). In order to incorporate the outstanding features into artificial aircrafts, we have to know how butterflies enhance aerodynamic forces, save power expenditure, and control their attitude while flying. 
One of interesting features of butterflies is the shape of their wings. A variety of studies in biology have investigated the relationship between the wing shape and the flight behavior in butterflies. Betts \& Wootton (1988) observed free flights of various butterfly species by using a high-speed camera, and analyzed the geometrical parameters of wing shapes and the kinematic parameters in representative flight modes. The analyses of these parameters suggested that butterflies with short broad (low aspect ratio) wings tend to fly slowly but to have high agility, whereas those with long slender (high aspect ratio) wings tend to fly fast and to use gliding extensively. The strong correlation between the wing shape and the flapping frequency has been studied in more detail by several researchers (Srygley 1999, Kingsolver 1999). Srygley (1999) compared the morphology and kinematics of four Heliconius species, which comprised two mimicry pairs containing two distinct lineages. It was found that the wing shape and the flapping frequency converged not within lineages but within mimicry groups. This result suggested that similar wing shapes result in similar flight behavior. Kingsolver (1999) artificially reduced the wing area of western white butterflies (Ponita occidentalis), and found that the reductions of the wing area significantly increased the flapping frequency of hovering butterflies in the laboratory experiments. In addition, the correlation between the wing shape and the acceleration capacity has been investigated for butterflies tethered to a needle (Berwaerts, van Dyck \& Aerts 2002) and for butterflies in take-off (Berwaerts, Matthysen \& van Dyck 2008). As a result, it was found that the aspect ratios of their forewings were positively related to the acceleration capacity in males of speckled wood butterflies (Pararge aegeria), although there were different trends between male and female.

Although the above studies have revealed the strong relationships between the wing shape and the flight behavior in butterflies, few researchers have investigated the effect of wing shape on the aerodynamic performance such as force, torque, and power expenditure from a viewpoint of physics. Recently, Ancel, Eastwood, Vogt, Ithier, Smith, Wood \& Kvač (2017) evaluated aerodynamic forces for various butterfly wing shapes and wing orientations both in experiments and numerical simulations using a low-speed wind tunnel. However, they considered only the gliding flight of butterflies, i.e., the relationship between the wing shape and the aerodynamic performance in their flapping flight was not investigated. On the other hand, Suzuki \& Yoshino (2018) numerically investigated the effect of wing shape (planform) on the aerodynamic performance in the flapping flight of a simple butterfly model composed of two thin rigid wings and a rod-shaped body (Suzuki, Minami \& Inamuro 2015). This model flaps downward to generate lift force and backward to generate thrust force like an actual butterfly. In this study, the aerodynamic performance was calculated for various wing planforms such as rectangular wings, trapezoidal wings, triangular wings, and an actual butterfly's wing. As a result, it was found that an actual butterfly's wing can generate aerodynamic forces more efficiently than the other wing planforms.

From a viewpoint of engineering, it is important to know how to design efficient wing planforms. In ordinary aircrafts with fixed wings, the effect of wing planform on 
aerodynamic performance is mainly governed by three geometrical parameters: span, chord, and sweep (Barbarino, Bilgen, Ajaj, Friswell \& Inman 2011). In non-dimensional forms, these parameters are likely to be represented by aspect ratio, taper ratio, and sweepback angle. On the other hand, such geometrical parameters in the flapping flight of butterflies have not been clarified. This is because the wing planforms of butterflies are so complex that they cannot be represented by only a few parameters. In designing butterfly-like MAVs, however, it would be very convenient if aerodynamic performance could be governed by a few geometrical parameters like ordinary aircrafts. In the present study, therefore, we attempt to identify a few geometrical parameters which can reproduce not the butterfly's wing itself but the efficient aerodynamic performance of the butterfly's wing. It should be noted that we focus on not gliding flight but flapping flight since the outstanding agility and maneuverability are likely to be caused by flapping motion.

We can find a clue for the above attempt from our previous parametric study using a simple butterfly model with rectangular or trapezoidal wings (Suzuki \& Yoshino 2018). In this study, the aerodynamic performance factors such as the lift force, the thrust force, the power expenditure, and the power loading were calculated for rectangular wings with various aspect ratios (ratio of the square of the wing length to the wing area) and for trapezoidal wings with various taper ratios (ratio of the wing-tip length to the wing-root length) when the wing area and the flapping frequency are fixed. As a result, it was found that the lift and thrust forces increase at the cost of the power expenditure as the taper ratio increases and as the aspect ratio increases. This is because wings with long wing-tip lengths can generate large wing-tip vortices (WTVs), which are regarded as a major source of the lift generation by a butterfly, (Yokoyama, Senda, Iima \& Hirai 2013) and wings with long wing lengths can generate strong WTVs due to a high flapping speed at the wing tips. In addition, it was found that the position of the rotational axis for the geometric angle of attack (AOA) can significantly affect the aerodynamic performance. For example, in the case when the rotational axis for the geometric AOA is located near the leading edge, the lift and thrust forces can be enhanced due to the kicking-down of the trailing edge like a dolphin kick. From these results, the reason why an actual butterfly's wing planform has high efficiency was explained that the aspect ratio is moderate, the taper ratio is effectively less than 1 , and the position of the rotational axis for AOA is located near the leading edge. Therefore, the aspect ratio, the taper ratio, and the position of the rotational axis for the geometric AOA can be regarded as potential candidates of governing geometrical parameters.

Another clue can be found from the investigation into the effect of wing orientation by Ancel et al. (2017). In their study, it was found that the wing orientation, i.e., the angle of the leading edge relative to the air flow, has a significant effect on the lift-drag ratio in gliding flight. Since the wing orientation can be represented by the sweepback angle, it might be a candidate of governing geometrical parameters even in flapping flight. 
From the above clues, we make a hypothesis that the efficient aerodynamic performance of the butterfly's wing can be reproduced by the following four geometrical parameters of wing planform: aspect ratio, taper ratio, position of the rotational axis for the geometric AOA, and sweepback angle. If this hypothesis is correct, a trapezoidal wing planform equivalent to an actual butterfly's wing planform in terms of aerodynamic performance must exist, since these four parameters can be defined in trapezoidal wing planforms. In order to test this hypothesis, therefore, we explore such a trapezoidal wing planform in the parameter space consisting of these four parameters. In the present study, we use the butterfly model used in our previous study (Suzuki \& Yoshino 2018), and calculate the aerodynamic performance of the model to find such a trapezoidal wing planform. In addition, we compare the flight behavior for a resulting trapezoidal wing planform with that for the actual butterfly's wing planform in the free flight of the model.

The paper is organized as follows. In section 2, we present the butterfly model and define the geometrical parameters of trapezoidal wing planforms. In section 3, we explain the governing equations and parameters of the system. The computational parameters are presented in section 4 , and results and discussion are shown in section 5 . We finally conclude in section 6 .

\section{Butterfly model}

\subsection{Outline}

The butterfly model used in this paper and its wing motion are illustrated in figure 1. It flaps downward and backward to generate lift and thrust forces, respectively. The motions of the left and right wings are symmetrical with respect to the longitudinal plane. The wing motion is a combination of a flapping motion and an attacking motion, described by the flapping angle $\theta$ and the geometric angle of attack (AOA)

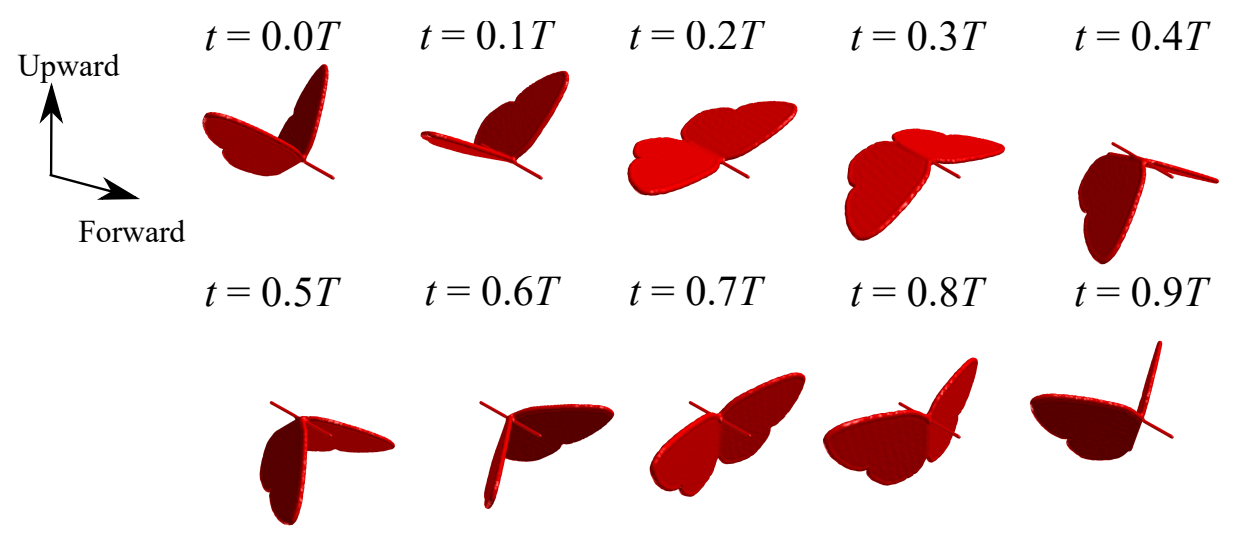

Figure 1. Illustration of a simple butterfly model and its wing motion during one period. The actual butterfly's wing planform is displayed in this figure as an example. This figure is taken from Suzuki \& Yoshino (2018). 
$\alpha$, respectively. The flapping angle $\theta(t)$ and the geometric AOA $\alpha(t)$ at time $t$ are given as follows:

$$
\begin{aligned}
& \theta(t)=\theta_{\mathrm{m}} \cos \left(\frac{2 \pi}{T} t\right), \\
& \alpha(t)=\frac{\alpha_{\mathrm{m}}}{2}\left[1+\cos \left(\frac{2 \pi}{T} t+\gamma\right)\right],
\end{aligned}
$$

where $\theta_{\mathrm{m}}$ is the flapping amplitude corresponding to half the stroke amplitude, $\alpha_{\mathrm{m}}$ is the maximum geometric AOA, $T$ is the period of flapping motion, and $\gamma$ is the phase shift. In this study, we set $\theta_{\mathrm{m}}=45^{\circ}, \alpha_{\mathrm{m}}=90^{\circ}$, and $\gamma=\pi / 2$, which is almost the optimal set of parameters for the butterfly model in terms of aerodynamic performance (Suzuki \& Yoshino 2017). It should be noted that the wing motion is the same as that of the model in Suzuki et al. (2015). For more details about the wing kinematics of the model, see Suzuki et al. (2015).

Although the wing kinematics of the present model are given as simple harmonic functions, those of actual butterflies are more complex (Sunada, Kawachi, Watanabe \& Azuma 1993, Yokoyama et al. 2013, Fei \& Yang 2015). However, the flapping angle and the geometric AOA of an actual butterfly can be approximated by simple harmonic functions without damaging the rough shapes of the curves of these angles (Fei \& Yang 2015). Also, in terms of the kinematic parameters $\left(\theta_{\mathrm{m}}, \alpha_{\mathrm{m}}, \gamma\right)$, the wing kinematics of the present model are artificial, since these parameters are determined so that the efficiency (power-loading coefficient) is almost maximum (Suzuki \& Yoshino 2017). Unfortunately, appropriate kinematic parameters have not been known, since they might significantly depend on species and flight modes (e.g., take-off, climbing flight, forward flight, and gliding). In the present study, therefore, we consider the situation where a butterfly flies most efficiently. It should be noted that we do not consider the clap-andfling motion (Weis-Fogh 1973) in the present study, although a butterfly can choose to use (or not to use) this motion depending on the flight mode (Srygley \& Thomas 2002).

In the present study, we explore a trapezoidal wing planform equivalent to an actual butterfly's wing planform in terms of aerodynamic performance in the parameter space consisting of the following four parameters: aspect ratio, taper ratio, position of the rotational axis for the geometric AOA, and sweepback angle. We assume that all the wing planforms used in this paper have the common wing area $S$ per one wing. We define the characteristic length by $L_{\mathrm{ref}}=\sqrt{S}$. The wings are infinitely thin and rigid. It must be mentioned that the aerodynamic performance depends on wing flexibility and twist (Zhang, Hedrick \& Mittal 2013) as well. In addition, the wing inertia affects the wing flexibility and twist (Combes \& Daniel 2003), and consequently affects the aerodynamic performance. In this paper, however, we focus on the wing shape, and the wing flexibility and twist are beyond the scope of the present study. In addition, we do not take the effect of the wing inertia into account, i.e., the wing mass is set to be zero.

The body of the model is a thin rod with length equal to $L_{\text {ref. }}$ The wings are connected to the mid-point of the body. The rotational axis for the geometric AOA is perpendicular to the body, and that for the flapping angle is parallel to the body. 


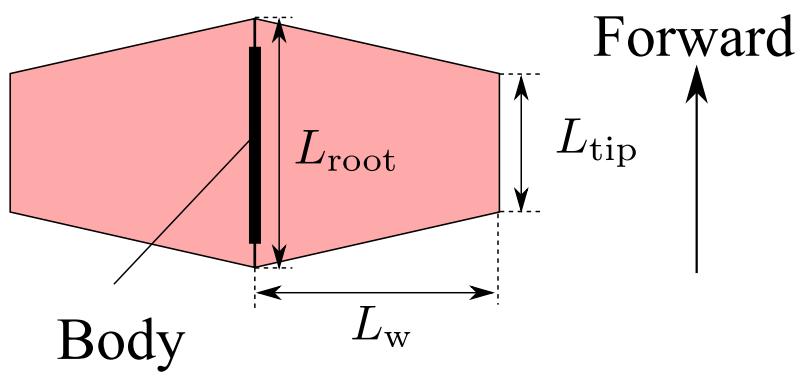

Figure 2. A trapezoidal wing planform. It should be noted that while the body of the model is infinitely thin, it is depicted with a finite thickness in this figure for ease of seeing.

It should be noted that the body has a negligible effect on the flow field and the aerodynamic forces acting on the model. When we simulate the free flight of the model, we use a body composed of two parts, i.e., thorax and abdomen, like an actual butterfly to control its attitude (see section 5.2).

\subsection{Trapezoidal wing planform}

We consider trapezoidal wing planforms with the wing area $S$. Let the wing length, the wing-tip length, and the wing-root length be $L_{\mathrm{w}}, L_{\text {tip }}$, and $L_{\text {root }}$, respectively, as shown in figure 2. Since the wing area is fixed at $S$, we obtain the following equation:

$$
S=\frac{1}{2}\left(L_{\text {tip }}+L_{\text {root }}\right) L_{\mathrm{w}} .
$$

\subsubsection{Aspect ratio and taper ratio}

The aspect ratio $A R$ and the taper ratio $T R$ for trapezoidal wing planforms are defined as follows:

$$
\begin{aligned}
A R & =\frac{L_{\mathrm{w}}^{2}}{S}, \\
T R & =\frac{L_{\mathrm{tip}}}{L_{\mathrm{root}}} .
\end{aligned}
$$

When we set the values of $A R$ and $T R$, the lengths $L_{\mathrm{w}}, L_{\text {tip }}$, and $L_{\text {root }}$ are determined as a function of $S$ from (3)-(5). In the wing planforms with $A R>1$, the wing length $L_{\mathrm{w}}$ is larger than the average wing-chord length $L_{\mathrm{c}}=\left(L_{\mathrm{tip}}+L_{\text {root }}\right) / 2$, and vice versa. The wing planforms with $T R<1$ correspond to taper wings, and those with $T R>1$ correspond to inverse-taper wings.

\subsubsection{Position of the rotational axis for the geometric $A O A$}

The rotational axis for the geometric AOA is the axis which is perpendicular to the body and passes through the connection point between the body and the wings. The position of the rotational axis for the geometric AOA is illustrated in figure 3 . Let $\ell$ be the position of the rotational axis for the geometric AOA relative to the center of the 
wing root, where $\ell>0$ and $\ell<0$ mean that the rotational axis is located on the side close to the leading edge and the trailing edge, respectively. It should be noted that the wing area $S$, the aspect ratio $A R$, and the taper ratio $T R$ are independent of $\ell$.

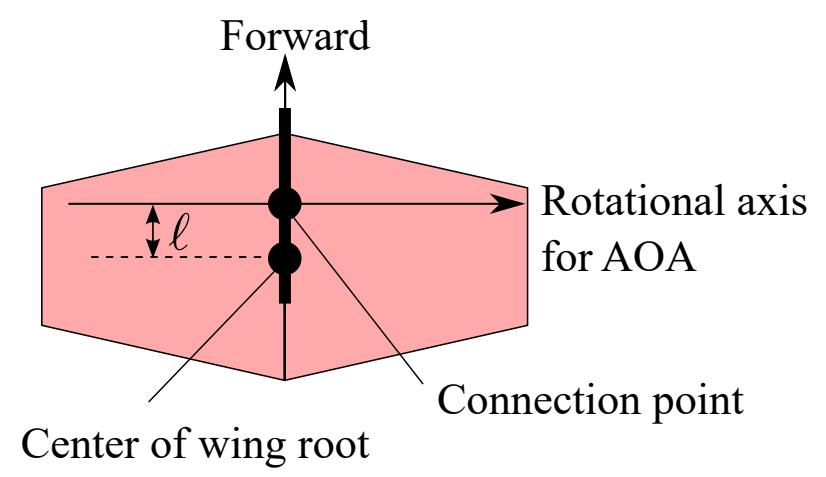

Figure 3. Rotational axis for the geometric angle of attack (AOA) shifted by $\ell$ relative to the center of the wing root.

\subsubsection{Sweepback angle}

The sweepback angle $\lambda$ is defined by the angle between a line perpendicular to the wing root and the line through the mod-points of the wing root and the wing tip as shown in figure 4 . We define that the wings have backward sweep and forward sweep when $\lambda>0$ and $\lambda<0$, respectively. It should be noted that the wing area $S$, the aspect ratio $A R$, the taper ratio $T R$, and the position $\ell$ of the rotational axis for the geometric AOA are independent of $\lambda$.

The sweepback angles of the leading and trailing edges are different from the abovedescribed $\lambda$, and they depend on $\lambda, L_{\text {tip }}, L_{\text {root }}$, and $L_{\mathrm{w}}$ as follows:

$$
\begin{aligned}
& \lambda_{\text {lead }}=\arctan \left(\tan \lambda-\frac{L_{\mathrm{tip}}-L_{\mathrm{root}}}{2 L_{\mathrm{w}}}\right), \\
& \lambda_{\text {trail }}=\arctan \left(\tan \lambda+\frac{L_{\mathrm{tip}}-L_{\mathrm{root}}}{2 L_{\mathrm{w}}}\right) .
\end{aligned}
$$

In the investigation into the effect of wing orientation on the gliding performance of butterfly wings by Ancel et al. (2017), the best performance was achieved when the leading edges of the wings are nearly perpendicular to the air flow. Therefore, it might be expected that such an orientation gives a good aerodynamic performance in flapping flight as well. In the present study, we define the standard sweepback angle as the angle which makes the leading edges of the wings are perpendicular to the forward direction. The standard sweepback angle $\lambda_{0}$ is given as follows:

$$
\lambda_{0}=\arctan \left(\frac{L_{\mathrm{tip}}-L_{\mathrm{root}}}{2 L_{\mathrm{w}}}\right) .
$$

It should be noted that when $\lambda=\lambda_{0}$, we can derive $\lambda_{\text {lead }}=0^{\circ}$ from Eqs. (6) and (8). 


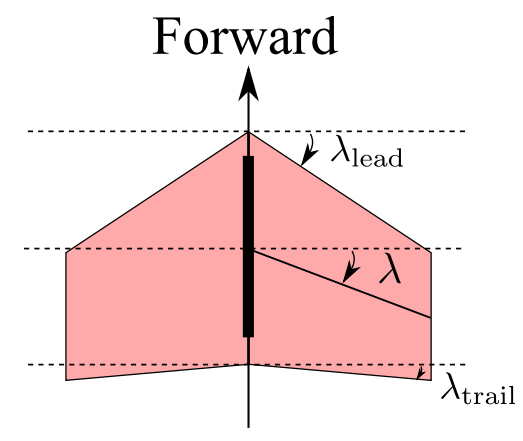

Figure 4. The sweepback angle $\lambda$, the leading edge sweepback angle $\lambda_{\text {lead }}$, and the trailing edge sweepback angle $\lambda_{\text {trail }}$.

\subsection{Actual butterfly's wing planform}

We explore a trapezoidal wing planform equivalent to an actual butterfly's wing planform in terms of aerodynamic performance. As the target wing planform, we use the wing planform of a small butterfly (Janatella leucodesma) used in our previous study (Suzuki \& Yoshino 2018). The wing planform is shown in figure 5. We assume that the area of the wing planform has the same wing area $S$ as the trapezoidal wing planforms.

As mentioned in Suzuki \& Yoshino (2018), the wing planform is constructed from a photograph of a J. leucodesma (Warren, Davis, Grishin, Pelham \& Stangeland accessed 2013-10-14). While an actual butterfly has fore-wings and hind-wings, i.e., totally four wings, we assume that the fore-wing and the hind-wing on each side coalesce together into one rigid plate, i.e., the model has totally two wings. The aspect ratio of this wing planform is $A R=1.61$. The connection point between the two wings and the body is set in a manner such that a point obtained by internally dividing the wing root by 0.0792 : 0.9208 (Warren et al. accessed 2013-10-14) is located at the middle point of the body. This means that the position of the rotational axis for the geometric AOA is at $\ell=0.36 c_{\max }$ where $c_{\max }=0.775 L_{\text {ref }}$ is the maximum chord length.

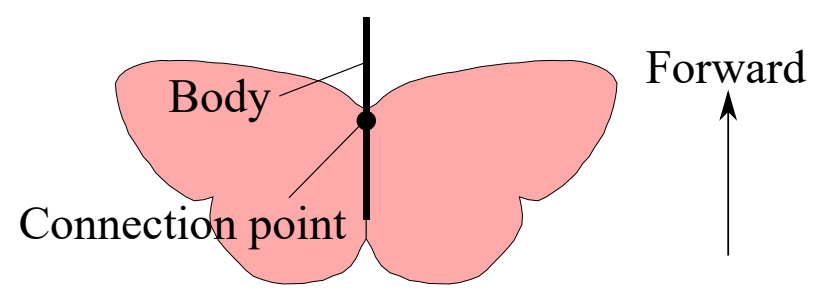

Figure 5. The wing planform of an actual butterfly (J. leucodesma). It should be noted that while the body of the model is infinitely thin, it is depicted with a finite thickness in this figure for ease of seeing. 


\section{Governing equations and non-dimensional parameters}

The fluid motion around the butterfly model is governed by the continuity equation and the Navier-Stokes equation for incompressible fluid as follows:

$$
\begin{aligned}
& \boldsymbol{\nabla} \cdot \boldsymbol{u}=0, \\
& \frac{\partial \boldsymbol{u}}{\partial t}+(\boldsymbol{u} \cdot \boldsymbol{\nabla}) \boldsymbol{u}=-\frac{1}{\rho_{\mathrm{f}}} \nabla p+\nu \nabla^{2} \boldsymbol{u},
\end{aligned}
$$

where $\boldsymbol{u}$ is the fluid velocity, $p$ is the pressure, $\rho_{\mathrm{f}}$ is the fluid density, and $\nu$ is the kinematic viscosity of the fluid. We consider the fluid to be air at room temperature $\left(20^{\circ} \mathrm{C}\right)$, and we set $\rho_{\mathrm{f}}=1.205 \mathrm{~kg} / \mathrm{m}^{3}$ and $\nu=1.512 \times 10^{-5} \mathrm{~m}^{2} / \mathrm{s}$. It should be noted that the gravitational term does not appear in (10). This is because the pressure $p$ includes the gravitational potential. The no-slip condition must be satisfied on the surface of the model, i.e., the fluid velocity must be equal to the velocity of the wings and the

body. It should be noted that the body has a negligible effect on the flow field and the aerodynamic forces acting on the model, although the no-slip condition is enforced on the body.

The governing parameter of the above equations (9) and (10) is the Reynolds number defined as follows:

$$
R e=\frac{U_{\mathrm{ref}} L_{\mathrm{ref}}}{\nu},
$$

where $U_{\text {ref }}$ is a characteristic flow speed and $L_{\text {ref }}$ is a characteristic length. As mentioned in section 2.1, we take $L_{\text {ref }}=\sqrt{S}$ where $S$ is the wing area. In this study, we consider the wing area $S=246.3 \mathrm{~mm}^{2}$ of J. leucodesma calculated from the data in Dudley (1990). In addition, we take $U_{\text {ref }}=4 \theta_{\mathrm{m}} L_{\text {ref }} / T$, which means the mean flapping speed of the reference point separated by $L_{\text {ref }}$ from the rotational axis for the flapping angle. It should be noted that the above definition of the Reynolds number is independent of the wing planforms, since all the wing planforms considered here have the same wing area $S$.

From a practical point of view, the same Reynolds number for the different wing planforms means the same flapping frequency $F_{\text {req }}=1 / T$. While the Reynolds number for a J. leucodesma calculated from the data in Dudley (1990) is $R e=892$ and that for other butterflies $R e \sim O\left[10^{3}\right]$, we set $R e=500$ in the present simulation. It is known that at the Reynolds number over several hundreds the effect of the Reynolds number on the aerodynamic performance is relatively insensitive for the butterfly model (Suzuki et al. 2015).

In this study, in order to explore a trapezoidal wing planform equivalent to the actual butterfly's wing planform in terms of aerodynamic performance, we calculate the aerodynamic performance when the body of the model is fixed. It should be noted that we assume that there is no forward velocity in this calculation. Then, we compare the flight behavior for the resulting trapezoidal wing planform with that for the actual butterfly's wing planform in the free flight of the model. In the free flight simulation, 
we have to consider the equations of motion of the model in addition to (9) and (10). For the detail of the equations of motion of the model, see section 5.2.

It should be noted that in this study the Strouhal number doesn't appear as a governing parameter unlike many other studies on flapping wings (e.g., Triantafyllou, Triantafyllou \& Grosenbaugh 1993, Wang 2000, Taylor, Nudds \& Thomas 2003). The Strouhal number is defined by $S t=F_{\text {req }} L_{\text {ref }} / U_{\text {ref }}$ where $F_{\text {req }}$ is the flapping frequency, $L_{\text {ref }}$ is the characteristic length, and $U_{\text {ref }}$ is the characteristic velocity. In general, the stroke amplitude and the forward velocity are chosen as $L_{\text {ref }}$ and $U_{\text {ref }}$, respectively (e.g., Shyy, Lian, Tang, Viieru \& Liu 2008). The Strouhal number is an important governing parameter when the forward velocity is chosen as the characteristic velocity. In this study, however, we assume that there is no forward velocity when we calculate the aerodynamic performance, and we use the flapping velocity as the characteristic velocity. Therefore, the Strouhal number is not included in governing parameters here. For the same reason, the reduced frequency (see Shyy et al. 2008, Shyy, Aono, Chimakurthi, Trizila, Kang, Cesnik \& Liu 2010) doesn't appear as a governing parameter. On the other hand, in the free flight simulation, we can calculate the Strouhal number by using the forward velocity of the model obtained as a resulting motion of the model. However, it is not a parameter but a result.

In this study, we calculate the following aerodynamic performance factors, i.e., the lift coefficient $C_{\mathrm{L}}$, the thrust coefficient $C_{\mathrm{T}}$, the pitching moment coefficient $C_{\mathrm{M}}$, and the power coefficient $C_{\mathrm{P}}$ :

$$
\begin{aligned}
C_{\mathrm{L}} & =\frac{F_{\text {lift }}}{0.5 \rho_{\mathrm{f}} U_{\text {ref }}^{2}(2 S)}, \\
C_{\mathrm{T}} & =\frac{F_{\text {thrust }}}{0.5 \rho_{\mathrm{f}} U_{\text {ref }}^{2}(2 S)}, \\
C_{\mathrm{M}} & =\frac{M_{\text {pitch }}}{0.5 \rho_{\mathrm{f}} U_{\text {ref }}^{2}(2 S) L_{\text {ref }}}, \\
C_{\mathrm{P}} & =\frac{\int_{\text {wing }} \boldsymbol{p}_{\text {local }} \cdot \boldsymbol{u}_{\text {local }} d S}{0.5 \rho_{\mathrm{f}} U_{\text {ref }}^{3}(2 S)},
\end{aligned}
$$

where $F_{\text {lift }}$ and $F_{\text {thrust }}$ are the lift and thrust forces acting on the model, i.e., the components of the aerodynamic force in the upward and forward directions, respectively, $M_{\text {pitch }}$ is the pitching moment acting on the model, $\boldsymbol{p}_{\text {local }}$ is the local stress acting on the fluid by a unit area of the wing surface, and $\boldsymbol{u}_{\text {local }}$ is the flow velocity at the point. Also, $\int_{\text {wing }} d S$ means the integral over the wings. Therefore, the power coefficient $C_{\mathrm{P}}$ represents the non-dimensional form of the power expenditure to move the wings against the aerodynamic force. It should be noted that the above definitions of the aerodynamic performance factors are also independent of the wing planforms, since all the wing planforms considered here have the same wing area $S$.

The time-averaged values of $C_{\mathrm{L}}, C_{\mathrm{T}}$, and $C_{\mathrm{P}}$ are important indices of the aerodynamic performance. Let the time-averaged values of $C_{\mathrm{L}}, C_{\mathrm{T}}$, and $C_{\mathrm{P}}$ in one 
stroke be $\overline{C_{\mathrm{L}}}, \overline{C_{\mathrm{T}}}$, and $\overline{C_{\mathrm{P}}}$. We define the power-loading coefficient as follows:

$$
C_{\mathrm{PL}}=\frac{\sqrt{{\overline{C_{\mathrm{L}}}}^{2}+{\overline{C_{\mathrm{T}}}}^{2}}}{\overline{C_{\mathrm{P}}}} \text {. }
$$

It should be noted that in this study the power-loading coefficient is defined by using the magnitude of the vector $\left(\overline{C_{\mathrm{T}}}, \overline{C_{\mathrm{L}}}\right)$, while in general it is defined by the ratio of the lift coefficient to the power coefficient. Since the present butterfly model generates the thrust force as well as the lift force, the above definition should be more appropriate as an index of efficiency.

\section{Numerical method and computational parameters}

The numerical method used in this study is the same as that in Suzuki et al. (2015); we use the IB-LBM approach (Suzuki \& Inamuro 2011) to solve (9) and (10). The

aerodynamic forces $F_{\text {lift }}$ and $F_{\text {thrust }}$ and the pitching moment $M_{\text {pitch }}$ are calculated by the summation of the body force which is applied to enforce the no-slip condition on the model in the immersed boundary method. In this calculation, the internal mass effect (see Suzuki \& Inamuro (2011)) is neglected, since the model has no volume. The wings and the body of the model are represented by an arrangement of boundary Lagrangian points. The position and velocity of the boundary Lagrangian points on the wings and the body are updated by orthogonal transformation of the coordinate systems fixed to the wings and the body relative to that fixed to the flight space. For details of the numerical method, see Suzuki \& Inamuro (2011). The verification of the numerical method and the convergence studies for temporal and spatial resolutions have been extensively checked in Suzuki et al. (2015) and Suzuki \& Yoshino (2018). In addition, Engels, Kolomenskiy, Schneider \& Sesterhenn (2016) simulated the same problem as Suzuki et al. (2015) to verify their proposed method (a Fourier method with volume penalization), and they reported that their result for the free flight of the butterfly model had a good agreement with that obtained in Suzuki et al. (2015).

The computational domain is the same as that used in Suzuki \& Yoshino (2018): we use a cuboid of $18 L_{\text {ref }} \times 12 L_{\text {ref }} \times 12 L_{\text {ref }}$. The $x-, y$-, and $z$-axes are fixed to the domain, and we denote the directions of the $x$ - and $y$-axes as forward and upward, respectively. The boundary condition on two sides perpendicular to the $x$-axis is the periodic boundary condition, and on the other sides the no-slip condition is used. The center of the body is fixed at the center of the domain. The domain is initially filled with a stationary fluid at uniform pressure. We use a multi-block grid (Inamuro 2012) in order to save computation time. The multi-block grid is composed of a fine grid with a lattice spacing $\Delta x$ and a coarse grid with $2 \Delta x$. The size of the inner fine grid is set to $4.5 L_{\text {ref }} \sin \theta_{\mathrm{m}} \times 4.5 L_{\text {ref }} \sin \theta_{\mathrm{m}} \times 4.5 L_{\text {ref }}$, in order to confine the butterfly model to the inner fine grid including a sufficient margin. In order to reduce the computational cost, we calculate one-half of the computational domain with the mirror boundary condition on the longitudinal plane which passes through the center of the domain and 
is perpendicular to $z$-axis. This implies that the flow field is assumed to be symmetrical with respect to the longitudinal plane. In the present simulations, we set the spatial and temporal resolutions to $L_{\mathrm{ref}}=60 \Delta x$ and $T=6000 \Delta t$ (where $\Delta x$ is a lattice spacing and $\Delta t$ is the time step), respectively.

\section{Results and discussion}

In this section, we explore a trapezoidal wing planform equivalent to the actual butterfly's wing planform in terms of aerodynamic performance in the parameter space consisting of the aspect ratio $A R$, the taper ratio $T R$, the position $\ell$ of the rotational axis for the geometric AOA, and the sweepback angle $\lambda$, when the body of the model is fixed. Then, we compare the flight behavior for the resulting trapezoidal wing planform with that for the actual butterfly's wing planform in the free flight of the model.

\subsection{Aerodynamic performance when the body of the model is fixed}

As mentioned in section 1, the reason why an actual butterfly's wing has high efficiency was explained that the aspect ratio is moderate, the taper ratio is effectively less than 1 , and the position of the rotational axis for the geometric AOA is located near the leading edge (Suzuki \& Yoshino 2018). In addition, it might be expected from the results by Ancel et al. (2017) that the sweepback angle which makes the leading edge of the wings perpendicular to the forward direction gives a good aerodynamic performance even in flapping flight. From these considerations, we explore in the parameter space $(A R, T R, \ell, \lambda)$ where $1 \leq A R \leq 2$ (the aspect ratio of the actual butterfly's wing is 1.61), $0 \leq T R \leq 1, \ell \geq 0$, and $0 \leq \lambda / \lambda_{0} \leq 1.25$ (the leading edge of the wings is perpendicular to the forward direction when $\lambda=1.0 \lambda_{0}$ ).

\subsubsection{Adjusting aspect ratio $A R$}

First, we adjust the aspect ratio $A R$ when the other parameters $(T R, \ell, \lambda)$ are fixed to $(1.0,0.0,0.0)$. Figure 6 shows the time variations of the lift coefficient $C_{\mathrm{L}}$, the thrust coefficient $C_{\mathrm{T}}$, the pitching moment coefficient $C_{\mathrm{M}}$, and the power coefficient $C_{\mathrm{P}}$ for $9.0 \leq t / T \leq 10$. It should be noted that the simulations are conducted until $t / T=20$, but the results during each stroke are almost the same after $t / T=9$. Thus, we present the results of the 10th period here. As seen in figure 6 (a), the positive lift is produced during downstroke and the negative lift force is produced during upstroke. This is attributed to the wing kinematics that the wings are flapped downward and backward during downstroke and upstroke, respectively. Similar phenomenon can be seen from other numerical results using more realistic butterfly models whose wing kinematics were derived directly from those of actual butterflies (Yokoyama et al. 2013, Fei \& Yang 2015).

We can see from figures 6 (a) and (b) that the positive peaks of $C_{\mathrm{L}}$ and $C_{\mathrm{T}}$ significantly increase with $A R$, while the phases of the peaks are almost independent from $A R$ in the range $1.0 \leq A R \leq 2.0$. This is attributed to the fact that the wing 
length $L_{\mathrm{w}}$ increases with $A R$. The flapping speed at the wing tip increases with $L_{\mathrm{w}}$, and therefore the wing-tip vortex (WTV), which is regarded as a large source of the lift generation by a butterfly (Yokoyama et al. 2013), is intensified. The intensified WTV is likely to be a cause of the lift and thrust enhancement. In addition, we can see that the positive peaks of $C_{\mathrm{L}}$ and $C_{\mathrm{T}}$ for the rectangular wing planform with $A R=1.6$ are comparable to those for the actual butterfly's wing planform, while there is a large phase difference between the results for the two wing planforms.

We can see from figure 6 (c) that the magnitude of $C_{\mathrm{M}}$ decreases with $A R$. This is because the average wing-chord length $L_{\mathrm{c}}$ decreases with $A R$, and consequently the wing surface points where the aerodynamic forces are applied come near the rotational axis of the geometric AOA. In addition, we can see that the magnitude of $C_{\mathrm{M}}$ for the actual butterfly's wing planform is much larger than that for the rectangular wing planforms.

We can see from figure $6(\mathrm{~d})$ that the magnitude of $C_{\mathrm{P}}$ significantly increases with $A R$. This is because the flapping speed at the wing tip as well as the aerodynamic forces increase with $A R$. In addition, we can see that the magnitude of $C_{\mathrm{P}}$ for the rectangular wing planform with $A R=1.4$ are comparable to those for the actual butterfly's wing

(a)

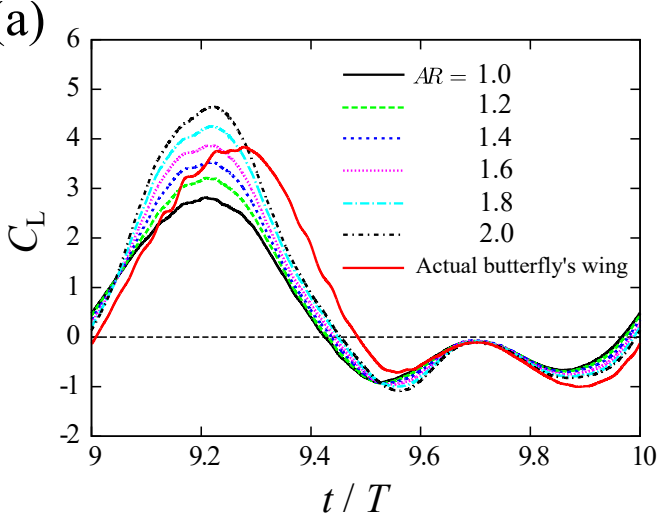

(c)

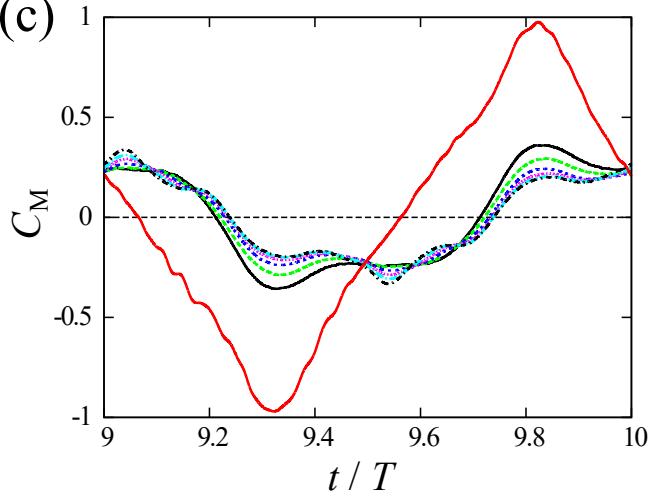

(b)

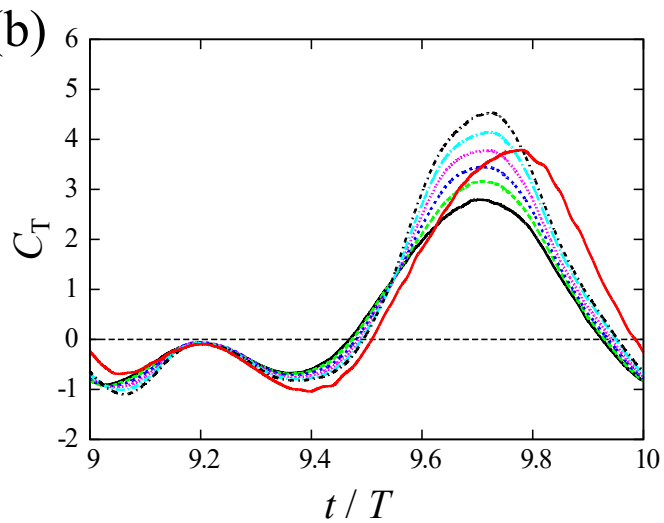

(d)

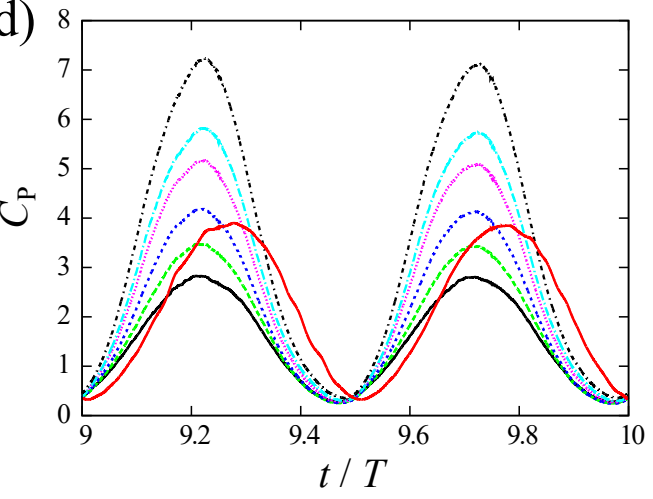

Figure 6. Time variations of (a) lift coefficient $C_{\mathrm{L}}$, (b) thrust coefficient $C_{\mathrm{T}}$, (c) pitching moment coefficient $C_{\mathrm{M}}$, and (d) power coefficient $C_{\mathrm{P}}$. The results of the trapezoidal wing planforms for various values of $A R$ when the other parameters $(T R, \ell, \lambda)$ are fixed to $(1.0,0.0,0.0)$ are compared with those of the actual butterfly's wing planform. 
planform, while there is a large phase difference between the results for the two wing planforms.

Since the parameter space is still large, we cannot determine the aspect ratio $A R$ only from the above results. In this study, we set $A R=1.6$, since this value is close to the aspect ratio of the actual butterfly's wing and makes the positive peaks of $C_{\mathrm{L}}$ and $C_{\mathrm{T}}$ comparable to those for the actual butterfly's wing planform.

\subsubsection{Adjusting taper ratio TR}

Second, we adjust the taper ratio $T R$ when the other parameters $(A R, \ell, \lambda)$ are fixed to $(1.6,0.0,0.0)$. We can see from figures 7 (a), (b), and (d) that the positive peaks of $C_{\mathrm{L}}$, $C_{\mathrm{T}}$, and $C_{\mathrm{P}}$ decrease as the value of $T R$ decreases, while the phases of the peaks are almost independent from $T R$ in the range $0.0 \leq T R \leq 1.0$. This is attributed to the fact that the wing-tip length $L_{\text {tip }}$ decrease as the value of $T R$ decreases. The WTV is likely to be smaller as $L_{\text {tip }}$ decreases, and therefore both the aerodynamic forces and the power expenditure decrease. In addition, we can see that the positive peaks of $C_{\mathrm{L}}$ and $C_{\mathrm{T}}$ for the trapezoidal wing planforms are smaller than those for the actual butterfly's wing
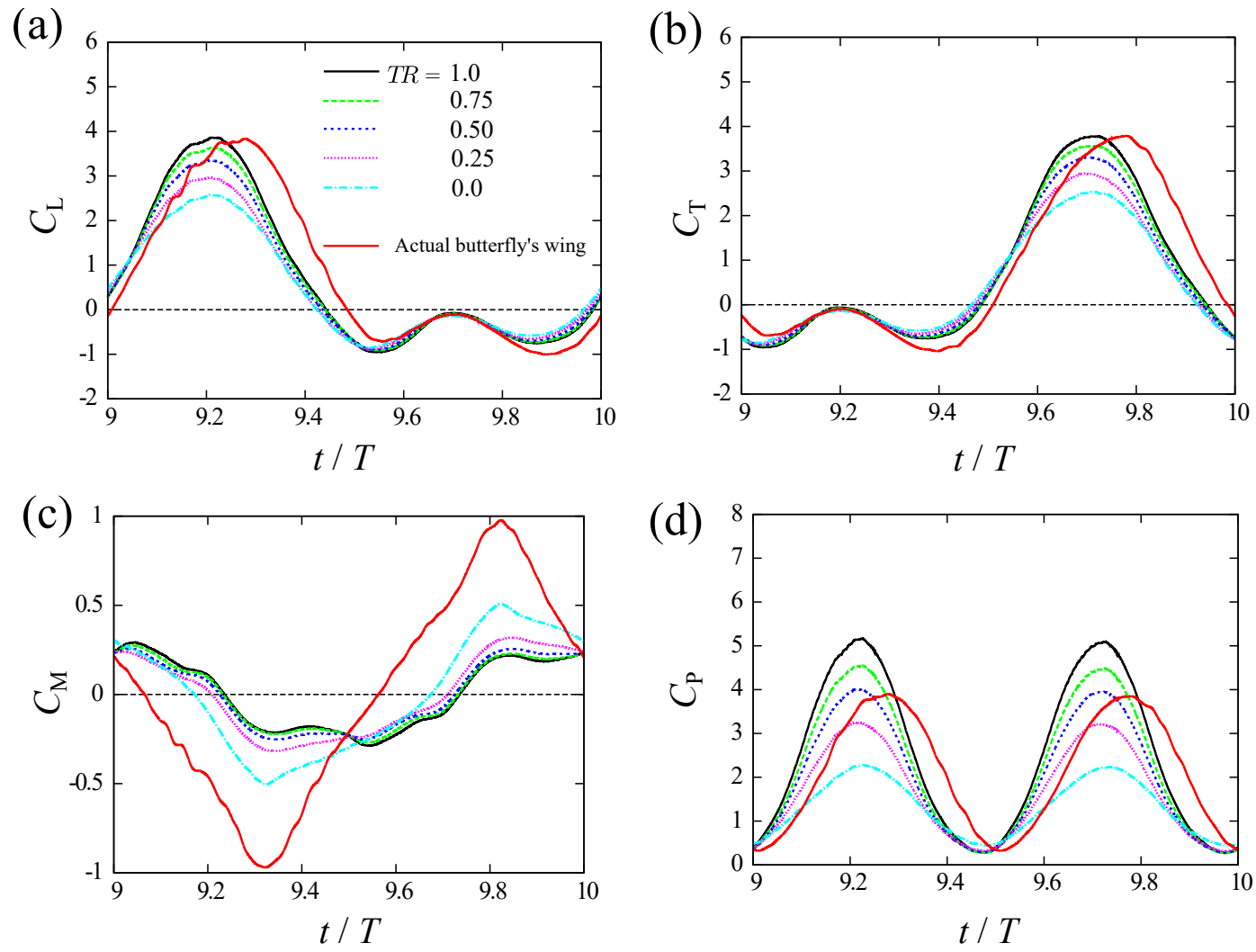

Figure 7. Time variations of (a) lift coefficient $C_{\mathrm{L}}$, (b) thrust coefficient $C_{\mathrm{T}}$, (c) pitching moment coefficient $C_{\mathrm{M}}$, and (d) power coefficient $C_{\mathrm{P}}$. The results of the trapezoidal wing planforms for various values of $T R$ when the other parameters $(A R, \ell, \lambda)$ are fixed to $(1.6,0.0,0.0)$ are compared with those of the actual butterfly's wing planform. 
planform, and the magnitude of $C_{\mathrm{P}}$ for the trapezoidal wing planform with $T R=0.50$ is comparable to that for the actual butterfly's wing planform.

On the other hand, we can see from figure 7 (c) that magnitude of $C_{\mathrm{M}}$ increases as the value of $T R$ decreases. This is because the wing surface points around the wing root become more distant from the rotational axis of the geometric AOA as the value of $T R$ decreases. However, the magnitude of $C_{\mathrm{M}}$ for the actual butterfly's wing planform is much larger than that for the trapezoidal wing planforms in the range $0.0 \leq T R \leq 1.0$.

From the above results, all the curves of $C_{\mathrm{L}}, C_{\mathrm{T}}, C_{\mathrm{M}}$, and $C_{\mathrm{P}}$ for the trapezoidal wing planforms are still far from those for the actual butterfly's wing planform. In this study, we set $T R=0.25$, since it can be expected from the results by Suzuki \& Yoshino (2018) that the positive peaks of $C_{\mathrm{L}}, C_{\mathrm{T}}$, and $C_{\mathrm{P}}$ increase when the position of the rotational axis for the geometric AOA is shifted forward.

\subsubsection{Adjusting position $\ell$ of the rotational axis for the geometric AOA}

Third, we adjust the position $\ell$ of the rotational axis for the geometric AOA when the other parameters $(A R, T R, \lambda)$ are fixed to $(1.6,0.25,0.0)$. Figure 8 shows the time variations of the aerodynamic performance factors for various values of $\ell$ normalized by

(a)
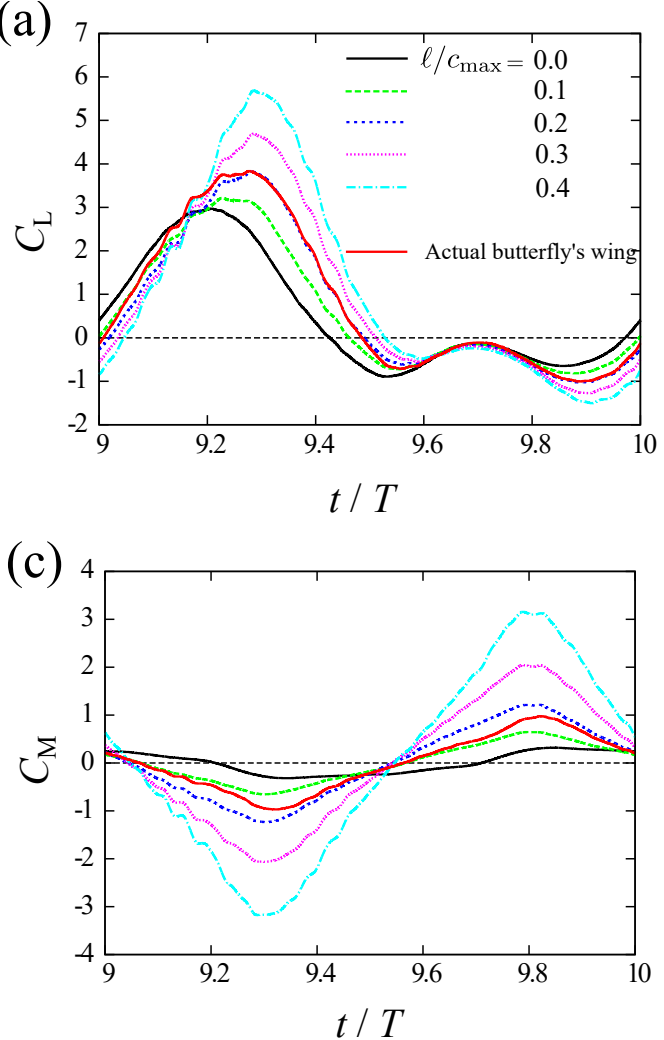

(b)

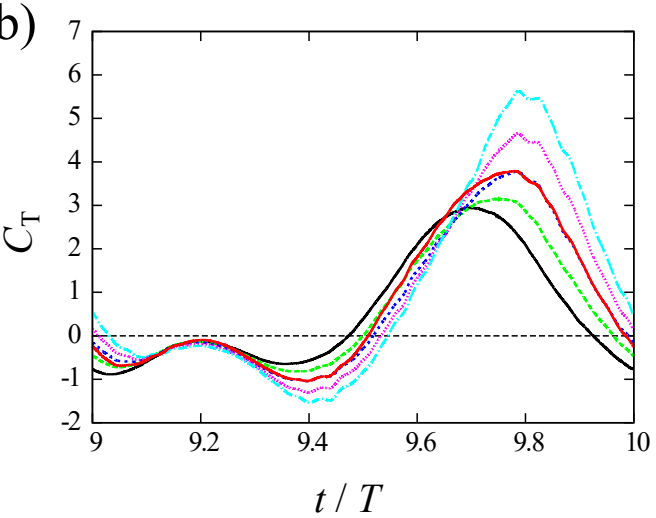

(d)

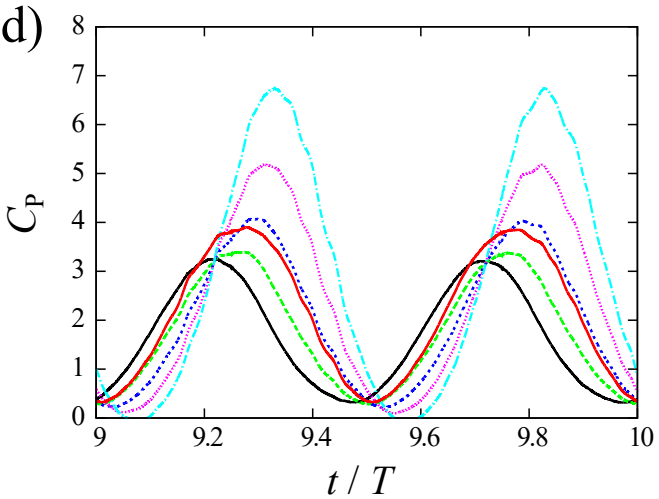

Figure 8. Time variations of (a) lift coefficient $C_{\mathrm{L}}$, (b) thrust coefficient $C_{\mathrm{T}}$, (c) pitching moment coefficient $C_{\mathrm{M}}$, and (d) power coefficient $C_{\mathrm{P}}$. The results of the trapezoidal wing planforms for various values of $\ell$ normalized by the maximum chord length $c_{\max }$ when the other parameters $(A R, T R, \lambda)$ are fixed to $(1.6,0.25,0.0)$ are compared with those of the actual butterfly's wing planform. 
the maximum chord length $c_{\max }$. It should be noted that $c_{\max }=L_{\text {root }}$ for the trapezoidal wing planform with $T R<1$. We can see from figures $8(\mathrm{a}),(\mathrm{b})$, and (d) that the positive peaks of $C_{\mathrm{L}}, C_{\mathrm{T}}$, and $C_{\mathrm{P}}$ increase with $\ell$, i.e., the position of the rotational axis comes near the leading edge, and the phases of these curves delay with $\ell$. This is attributed to the fact that the distance of the trailing edge from the rotational axis for the geometric AOA increases with $\ell$. This intensifies the effect of the kicking-down of the trailing edge like a dolphin kick, and this effect enhances the aerodynamic forces at the cost of the power expenditure and shifts these peak values (Suzuki \& Yoshino 2018).

We can see from figure 8 (c) that the magnitude of $C_{\mathrm{M}}$ increases with $\ell$. This is because the wing surface points around the trailing edge become more distant from the rotational axis of the geometric AOA as $\ell$ increases. In addition, we can see that the magnitude of $C_{\mathrm{M}}$ for the trapezoidal wing planforms with $\ell \geq 0.2 c_{\max }$ is larger than that for the actual butterfly's wing planform.

From the above results, we can see that the trapezoidal wing planform with $(A R, T R, \ell, \lambda)=\left(1.6,0.25,0.2 c_{\max }, 0.0\right)$ gives quite similar aerodynamic performance to the actual butterfly's wing planform. However, the magnitude of $C_{\mathrm{M}}$ for this trapezoidal

(a)

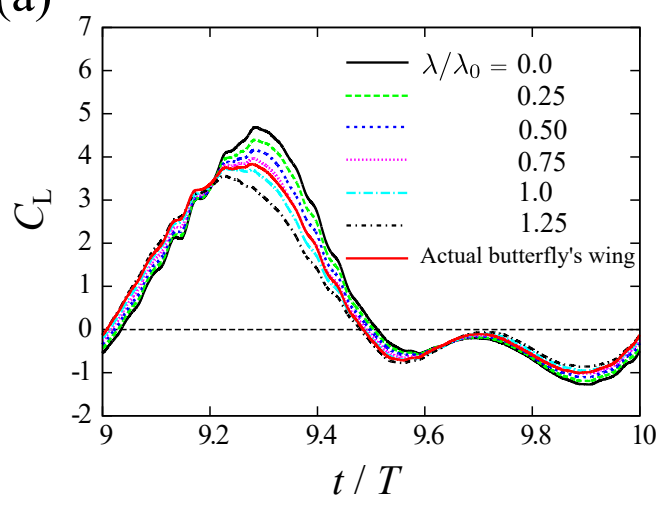

(c)

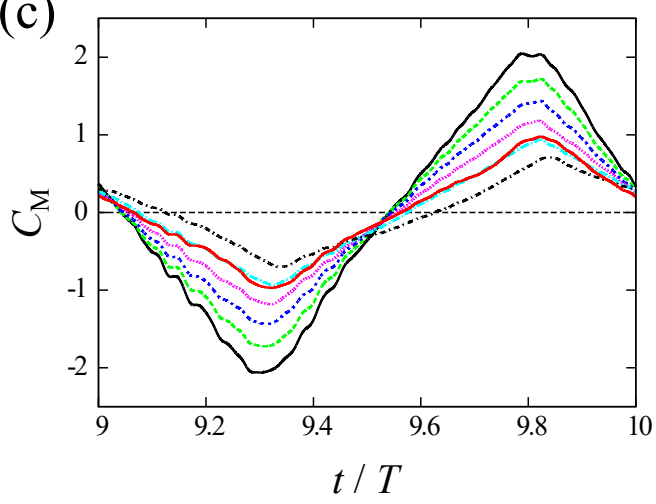

(b)

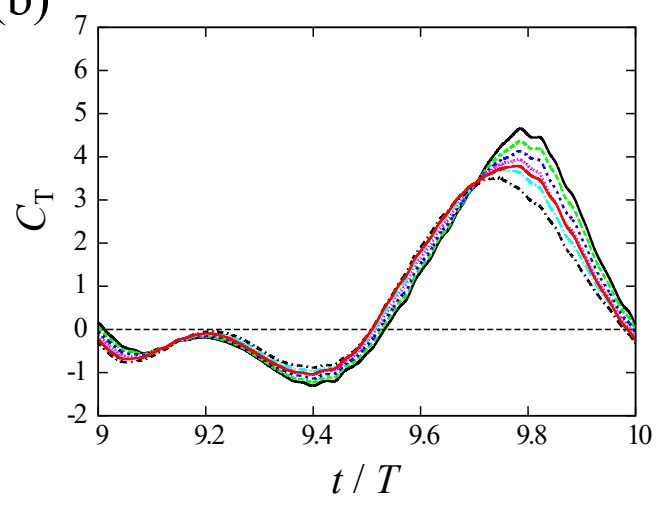

(d)

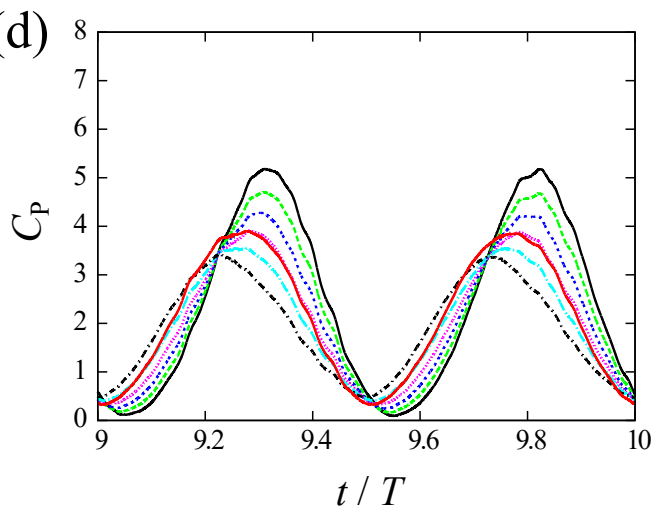

Figure 9. Time variations of (a) lift coefficient $C_{\mathrm{L}}$, (b) thrust coefficient $C_{\mathrm{T}}$, (c) pitching moment coefficient $C_{\mathrm{M}}$, and (d) power coefficient $C_{\mathrm{P}}$. The results of the trapezoidal wing planforms for various values of $\lambda$ when the other parameters $(A R, T R, \ell)$ are fixed to $\left(1.6,0.25,0.3 c_{\max }\right)$ are compared with those of the actual butterfly's wing planform. 
wing planform exceeds that for the actual butterfly's wing planform, and the curves of $C_{\mathrm{P}}$ still have a phase difference. In this study, we set not $\ell=0.2 c_{\max }$ but $\ell=0.3 c_{\max }$ in order to leave place for the effect of the sweepback angle $\lambda$.

\subsubsection{Adjusting sweepback angle $\lambda$}

Finally, we adjust the sweepback angle $\lambda$ when the other parameters $(A R, T R, \ell)$ are fixed to $\left(1.6,0.25,0.3 c_{\max }\right)$. Figure 9 shows the time variations of the aerodynamic factors for various $\lambda$ in the range $0 \leq \lambda / \lambda_{0} \leq 1.25$. This range of $\lambda$ is determined so that it includes the range where the leading edge sweepback angle $\lambda_{\text {lead }}$ is nearly equal to $0^{\circ}$. This comes from the expectation that when $\lambda_{\text {lead }} \simeq 0^{\circ}$, i.e., the leading edge of the wings is almost perpendicular to the forward direction, a good aerodynamic performance can be achieved even in flapping flight like the results by Ancel et al. (2017). It should be noted that from the definition of the standard sweepback angle (8), $\lambda_{0}$ is equal to $-20.56^{\circ}$ in this set of the parameters. This means that the wings have more forward sweep as $\lambda / \lambda_{0}$ increases. We can see from figure 9 that all the peaks of $C_{\mathrm{L}}, C_{\mathrm{T}}, C_{\mathrm{M}}$, and $C_{\mathrm{P}}$ decrease with $\lambda$. This is because the wing surface points around the leading edge come near the rotational axis of the geometric AOA as $\lambda$ increases, and consequently the kicking-down effect is weakened. As a result, we can see that the trapezoidal wing planform with $(A R, T R, \ell, \lambda)=\left(1.6,0.25,0.3 c_{\max }, \lambda_{0}\right)$ gives almost equivalent aerodynamic performance to the actual butterfly's wing planform.

\subsubsection{Butterfly-equivalent trapezoidal wing planform}

In summary, the resulting trapezoidal wing planform is shown in figure 10, and its geometrical parameters are shown in table 1 . Hereafter, we will call it butterfly-equivalent trapezoidal wing planform.

Figure 11 shows the locations of the centroids of the butterfly-equivalent trapezoidal wing planform and the actual butterfly's wing planform. We can see from this figure that the centroid of the former one is close to that of the latter one. This means that if the mass densities on these wings are assumed to be uniform and the same, the centers of mass of these wings are close to each other as well. Therefore, the inertial force and

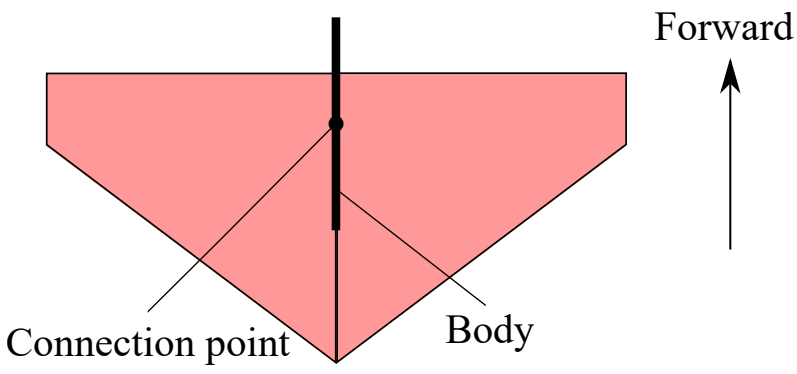

Figure 10. A trapezoidal wing planform equivalent to an actual butterfly's wing planform in terms of aerodynamic performance. It should be noted that while the body of the model is infinitely thin, it is depicted with a finite thickness in this figure for ease of seeing. 
Table 1. Parameters for a trapezoidal wing planform equivalent to an actual butterfly's wing planform in terms of aerodynamic performance, where $A R$ is the aspect ratio, $T R$ is the taper ratio, $\ell$ is the position of the rotational axis for the geometric AOA, $c_{\text {max }}$ is maximum chord length, $\lambda$ is the sweepback angle, and $\lambda_{0}$ is the standard sweepback angle.

\begin{tabular}{cccc}
\hline$A R$ & $T R$ & $\ell / c_{\max }$ & $\lambda$ \\
\hline 1.6 & 0.25 & 0.3 & $-20.56^{\circ}\left(=\lambda_{0}\right)$ \\
\hline
\end{tabular}

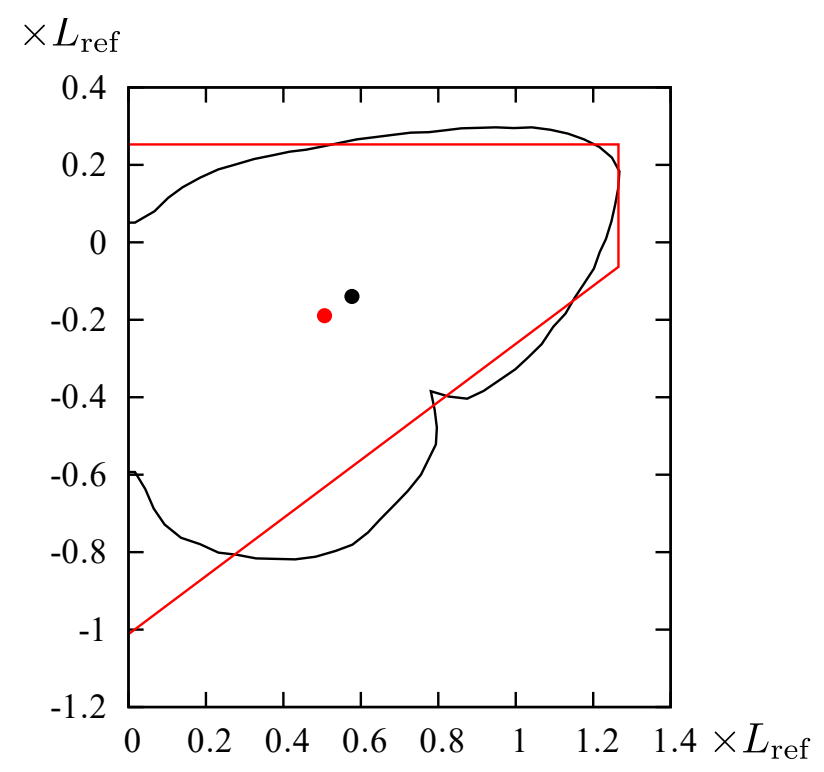

Figure 11. Locations of the centroids of the butterfly-equivalent trapezoidal wing planform (red) and the actual butterfly's wing planform (black). The outlines and centroids of these wing planforms are shown in solid lines and bullets, respectively, in the coordinate whose axes are normalized by the reference length $L_{\mathrm{ref}}$ and origin is located at the intersection point of the wing root and the rotational axis for the geometric AOA.

torque of these wings might be close to each other. In this paper, however, we focus on not the inertial force and torque but the aerodynamic force and torque generated by the wings, and the wing inertia is beyond the scope of the present study.

Figure 12 shows the time variation of the vortex structure near the butterfly model with the butterfly-equivalent trapezoidal wing planform and the actual butterfly's wing planform in the tenth period. The vortex structure is visualized by the Qcriterion (Hunt, Wray \& Moin 1988), i.e., the second invariant of the velocity gradient tensor given by

$$
Q=-\frac{\partial u_{i}}{\partial x_{j}} \frac{\partial u_{j}}{\partial x_{i}}
$$

where $i, j=x, y, z$ represent the Cartesian coordinates and the summation convention is used. We can see from figure 12 that the structures of the leading-edge vortex, the wing-tip vortex, and the separated vortices behind the model for the butterfly- 
(a)

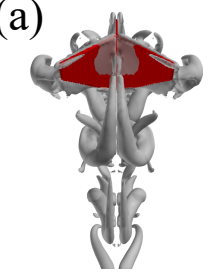

(b)

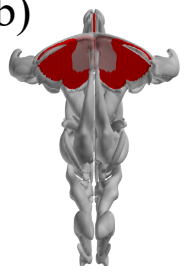

$t / T=9.00$
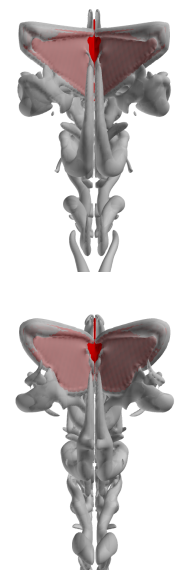

$t / T=9.17$
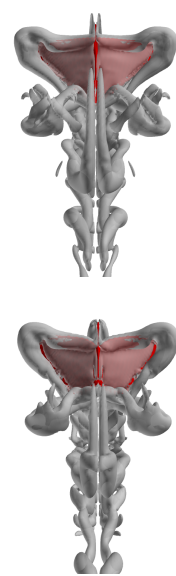

$t / T=9.33$
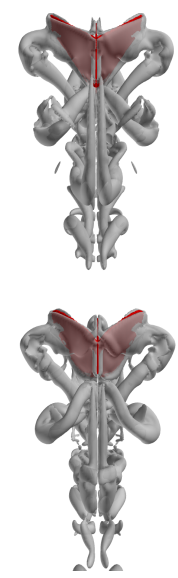

$t / T=9.50$
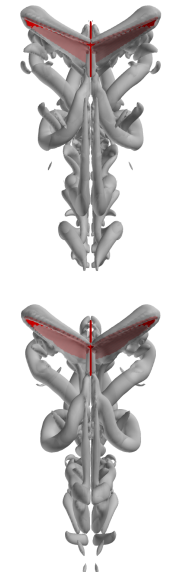

$t / T=9.67$

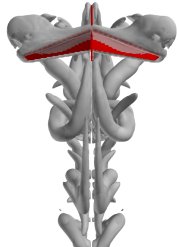

Forward

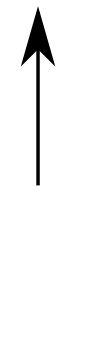

$t / T=9.83$

Figure 12. Vortex structures viewed from the upper side of the butterfly model: (a) the butterfly-equivalent trapezoidal wing planform and (b) the actual butterfly's wing planform. The model is shown in red, and the isosurface of $Q=3\left(U_{\text {ref }} / L_{\text {ref }}\right)^{2}$ is shown in gray.

equivalent trapezoidal wing planform are very similar to those for the actual butterfly's wing planform, although the geometries of the two wing planforms are largely different.

Figure 13 shows the time variations of the lift coefficient $C_{\mathrm{L}}$, the thrust coefficient $C_{\mathrm{T}}$, the pitching moment coefficient $C_{\mathrm{M}}$, and the power coefficient $C_{\mathrm{P}}$ for $9.0 \leq t / T \leq 10$. Although the results for $(A R, T R, \ell, \lambda)=\left(1.6,0.25,0.3 c_{\max }, \lambda_{0}\right)$ are included in figure 9 , we show them again in order to make it easy to compare the results of the butterflyequivalent trapezoidal wing planform with those of the actual butterfly's wing planform. We can see from figures $13(\mathrm{a})-(\mathrm{c})$ that the curves of $C_{\mathrm{L}}, C_{\mathrm{T}}$, and $C_{\mathrm{M}}$ for the two wing planforms are almost coincident with each other. In addition, we can see from figure $13(\mathrm{~d})$ that the peak value of $C_{\mathrm{P}}$ for the butterfly-equivalent wing planforms is slightly smaller than that for the actual butterfly's wing planform. This suggests that trapezoidal wing planforms with a good set of parameters can have a smaller power expenditure than the actual butterfly's wing, and consequently it is possible to construct more efficient trapezoidal wing planforms than the actual butterfly's wing.

Table 2 shows the time-averaged values of $C_{\mathrm{L}}, C_{\mathrm{T}}$, and $C_{\mathrm{P}}$ in the tenth period. In addition, the power-loading coefficient $C_{\mathrm{PL}}$ is shown in this table. It should be noted that the time-averaged value of $C_{\mathrm{M}}$ is not shown in this table, since it is more than two orders of magnitude smaller than the peak value, i.e., negligibly small. We can see from table 2 that $\overline{C_{\mathrm{L}}}, \overline{C_{\mathrm{T}}}$, and $\overline{C_{\mathrm{P}}}$ for the butterfly-equivalent wing planform are slightly smaller than those for the actual butterfly's wing planform. Consequently, in terms of the power-loading coefficient $C_{\mathrm{PL}}$, the efficiency of the two wing planforms is comparable.

The differences in the aerodynamic performance between the two wing planforms shown in figure 13 and table 2 are likely to come from the difference in the detailed shape (e.g., smoothness of the outline) between the two wing planforms. However, 
(a)
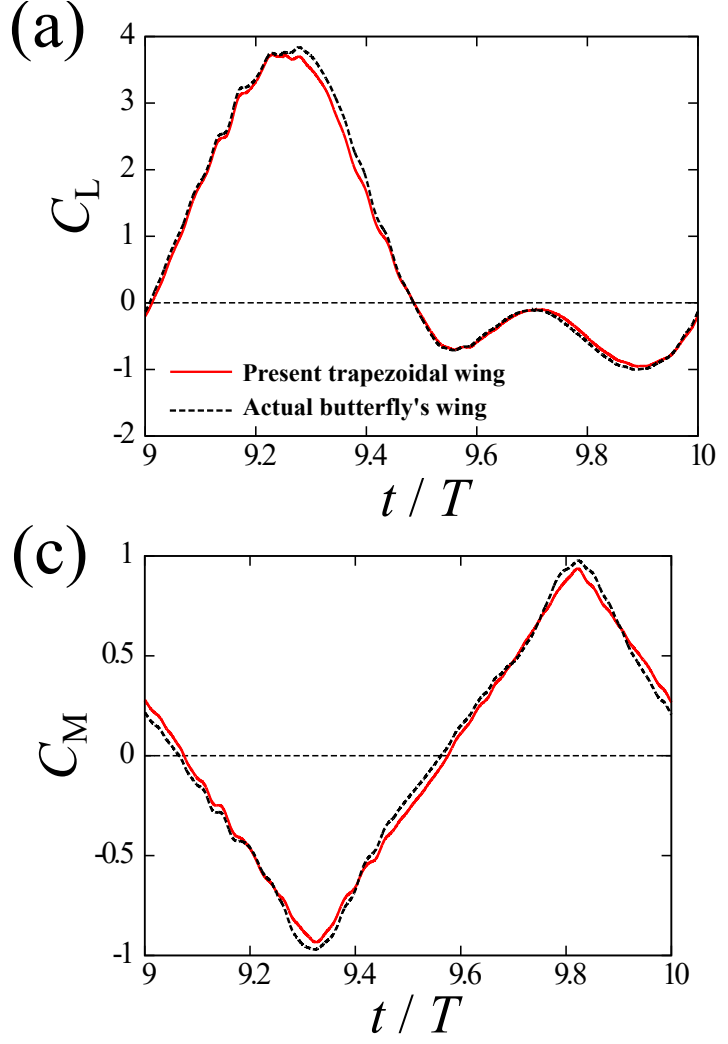

(b)

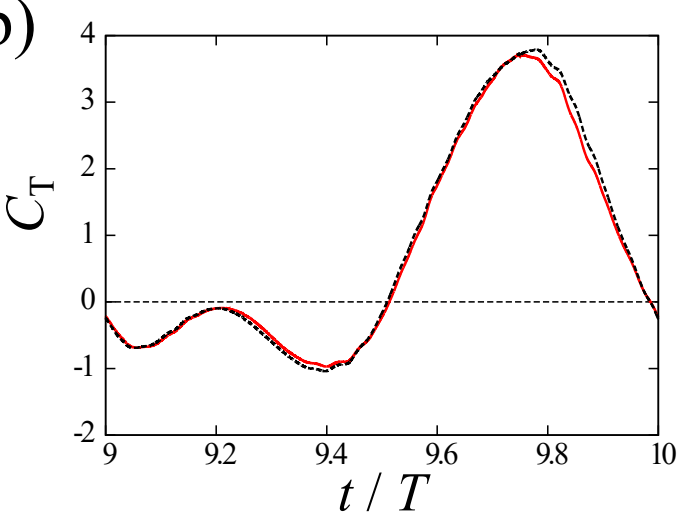

(d)

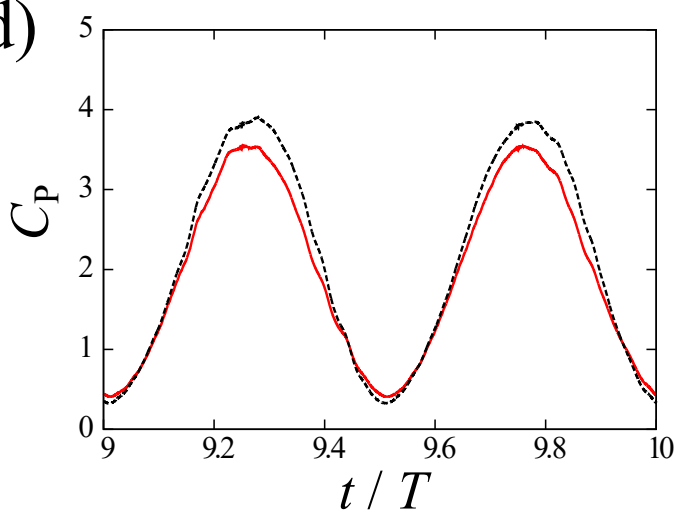

Figure 13. Time variations of (a) lift coefficient $C_{\mathrm{L}}$, (b) thrust coefficient $C_{\mathrm{T}}$, (c) pitching moment coefficient $C_{\mathrm{M}}$, and (d) power coefficient $C_{\mathrm{P}}$ for the butterflyequivalent trapezoidal wing planform and the actual butterfly's wing planform.

Table 2. The time-averaged lift coefficient $\overline{C_{\mathrm{L}}}$, the time-averaged thrust coefficient $\overline{C_{\mathrm{T}}}$, the time-averaged power coefficient $\overline{C_{\mathrm{P}}}$, and the power-loading coefficient $C_{\mathrm{PL}}$ for the butterfly-equivalent trapezoidal wing planform and the actual butterfly's wing planform.

\begin{tabular}{rrrrr}
\hline & $\overline{C_{\mathrm{L}}}$ & $\overline{C_{\mathrm{T}}}$ & $\overline{C_{\mathrm{P}}}$ & $C_{\mathrm{PL}}$ \\
\hline Present trapezoidal wing & 0.758 & 0.770 & 1.97 & 0.548 \\
Actual butterfly's wing & 0.812 & 0.794 & 2.13 & 0.533 \\
\hline
\end{tabular}

the differences in the aerodynamic performance are very small. This suggests that the four geometrical parameters $(A R, T R, \ell, \lambda)$ have a dominant effect on the aerodynamic performance for the present wing kinematics, and the detailed shape of the wing planform is not significant.

From the above results, we can find a trapezoidal wing planform equivalent to an actual butterfly's wing planform in terms of aerodynamic performance. This suggests that our hypothesis that the efficient aerodynamic performance of the butterfly's wing can be reproduced by $A R, T R, \ell$, and $\lambda$ is valid when the body of the model is fixed. 


\subsection{Free flight with pitching motion control}

Next, we compare the flight behavior for the butterfly-equivalent trapezoidal wing planform with that for the actual butterfly's wing planform in the free flight of the butterfly model. In this simulation, we calculate the free flight with pitching motion control. We use the control strategy used in Suzuki et al. (2015), i.e., we use a modified model whose body is composed of the thorax and the abdomen like an actual butterfly. Here, we assume that the body moves only in the $x$ (forward) and $y$ (upward) directions and rotates only in the pitching motion for simplicity.

It is known that the body of an actual butterfly is composed of the thorax and the abdomen, and the pitching angle of the body might be controlled by flexing at the joint between the thorax and the abdomen (Dudley 2002). As a simple model of the butterfly body, let the body of the model be composed of two straight infinitely-thin rods as shown in figure 14 (a): the thorax with the length $L_{\mathrm{t}}$ and the abdomen with the length $L_{\mathrm{a}}$. The total length of the body is $L_{\mathrm{b}}=L_{\mathrm{t}}+L_{\mathrm{a}}$. Let the mass of the thorax be $M_{\mathrm{t}}$ and that of the abdomen be $M_{\mathrm{a}}$. The total mass of the body is $M_{\mathrm{b}}=M_{\mathrm{t}}+M_{\mathrm{a}}$. The connection point between the thorax and the wings is located at the distance of $\ell_{0}$ from the head of the thorax. In this study, we set $L_{\mathrm{b}}=0.86 L_{\mathrm{ref}}, L_{\mathrm{t}}: L_{\mathrm{a}}=3: 7, M_{\mathrm{t}}: M_{\mathrm{a}}=44: 51$, and $\ell_{0}=0.77 L_{\mathrm{t}}$, which are the same as a J. leucodesma (Dudley 1990). We assume that the thorax and the abdomen are connected by a rotary actuator with no mass and no rotation friction.

We control the pitching angle of the thorax $\theta_{\mathrm{t}}$ by the input torque $T^{\mathrm{cont}}$ produced by the rotary actuator. In the same way as Suzuki et al. (2015), we determine the input torque $T^{\text {cont }}$ by the proportional-plus-integral-plus-derivative (PID) control as follows:

$$
T^{\mathrm{cont}}(t)=K_{\mathrm{p}}\left(\theta_{0}(t)-\theta_{\mathrm{t}}(t)\right)+K_{\mathrm{i}} \int_{0}^{t}\left(\theta_{0}\left(t^{\prime}\right)-\theta_{\mathrm{t}}\left(t^{\prime}\right)\right) d t^{\prime}+K_{\mathrm{d}}\left(\dot{\theta}_{0}(t)-\dot{\theta}_{\mathrm{t}}(t)\right),(18
$$

where $\theta_{0}$ is the target pitching angle of the thorax, $K_{\mathrm{p}}$ is the proportional gain, $K_{\mathrm{i}}$ is the integral gain, and $K_{\mathrm{d}}$ is the derivative gain. In order to suppress the increase in the pitching angle $\theta_{\mathrm{t}}$, we set $\theta_{0}(t)=0^{\circ}$.

The equations of motion of the model are the same as those in Suzuki et al. (2015), and we calculate them by the second-order Adams-Bashforth method in the same way as Suzuki et al. (2015). The governing parameters of these equations are the Froude number $F r$ and the non-dimensional mass $N_{\mathrm{M}}$ as follows:

$$
\begin{aligned}
& F r=\frac{U_{\text {ref }}}{\sqrt{L_{\mathrm{ref}} G}}, \\
& N_{\mathrm{M}}=\frac{M_{\mathrm{b}}}{\rho_{\mathrm{f}} L_{\mathrm{ref}}^{3}},
\end{aligned}
$$

where $G=9.807 \mathrm{~m} / \mathrm{s}^{2}$ is the gravitational acceleration.

Totally, the governing parameters of this system are the Reynolds number $R e$, the Froude number $F r$, and the non-dimensional mass $N_{\mathrm{M}}$. For an actual butterfly (J. leucodesma), these parameters are $\left(\operatorname{Re} F r, N_{\mathrm{M}}\right)=(892,2.19,5.16)$ (Dudley 1990). 
However, we use $\left(\operatorname{Re} F r, N_{\mathrm{M}}\right)=(300,8.815,38)$, which are comparable to the parameters for a fruit fly rather than a butterfly, in order to reduce the computational cost. In addition, we set $K_{\mathrm{p}}=-M_{\mathrm{a}} L_{\mathrm{a}}^{2} F_{\text {req }}^{2} \times 0.698, K_{\mathrm{i}}=-M_{\mathrm{a}} L_{\mathrm{a}}^{2} F_{\text {req }}^{3} \times 0.698$, and $K_{\mathrm{d}}=-M_{\mathrm{a}} L_{\mathrm{a}}^{2} F_{\text {req }} \times 9.65$, which are the same as used in Suzuki et al. (2015).

Figures 14 (b)-(d) show the trajectory of the center of the thorax, the time variation of the pitching angle $\theta_{\mathrm{t}}$ of the thorax, and the time variation of the relative angle $\psi$ of the abdomen to the thorax. We can see from these figures that the results for the two wing planforms are comparable with each other. Therefore, the butterfly-equivalent trapezoidal wing planform has almost the same flight behavior as the actual butterfly's wing planform. This suggests that our hypothesis that the efficient aerodynamic performance of the butterfly's wing can be reproduced by $A R, T R, \ell$, and $\lambda$ is valid even in the free flight with pitching motion control.

(a)

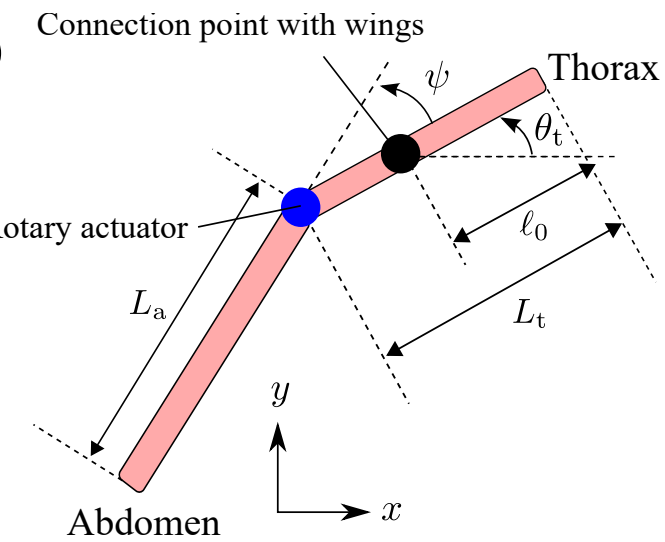

(c)

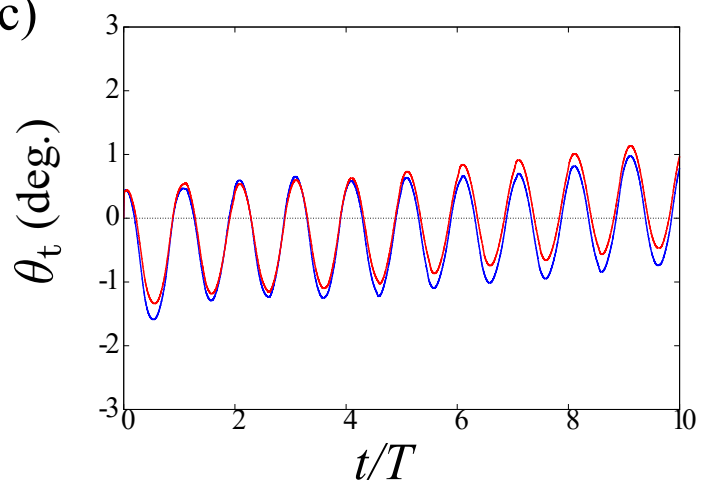

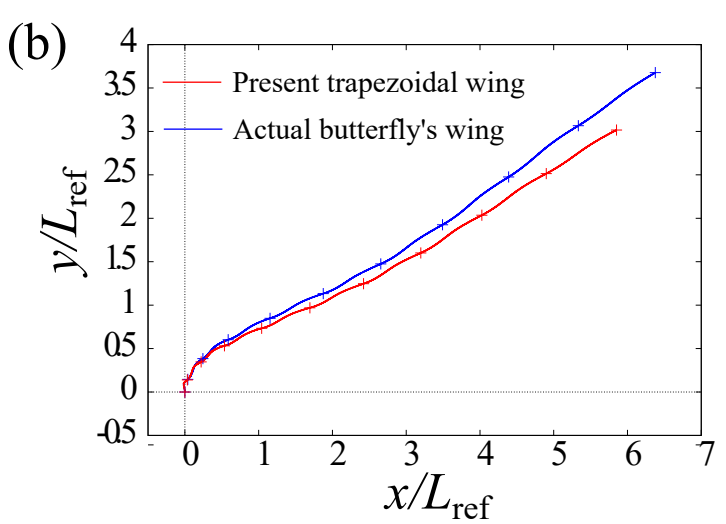

(d)

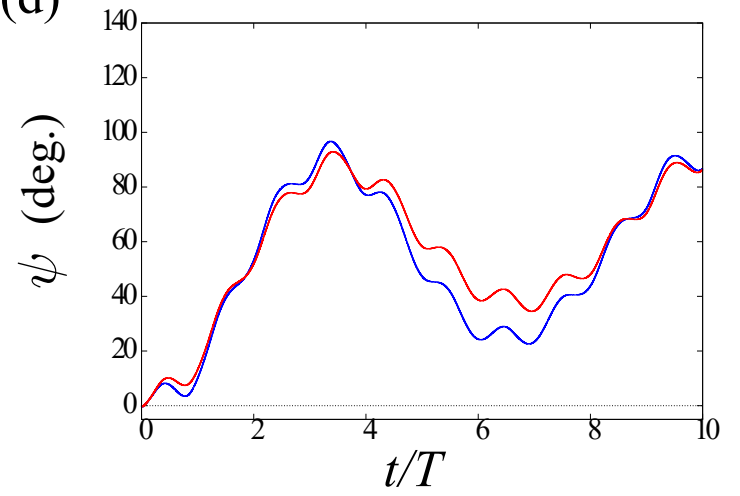

Figure 14. (a) The body composed of the thorax and the abdomen to control the pitching angle of the butterfly model, (b) trajectory of the center of the thorax, (c) time variation of the pitching angle $\theta_{\mathrm{t}}$ of the thorax, and (d) time variation of the relative angle $\psi$ of the abdomen to the thorax when the pitching angle of the thorax is controlled by flexing the abdomen. In (b), the initial position of the center of the thorax is $\left(x / L_{\text {ref }}, y / L_{\text {ref }}\right)=(0,0)$, and the symbols on the trajectories indicate the position of the center of the thorax when the wings are at $\theta=\theta_{\mathrm{m}}$. 


\section{Conclusions}

We made a hypothesis that the efficient aerodynamic performance of the butterfly's wing can be reproduced by the following four geometrical parameters of wing planform: aspect ratio, taper ratio, position of the rotational axis for the geometric AOA, and sweepback angle. In order to test this hypothesis, we explored a trapezoidal wing planform equivalent to an actual butterfly's wing planform in terms of aerodynamic performance in the parameter space consisting of these four parameters through immersed boundarylattice Boltzmann simulations of the flapping flight of a simple butterfly model. As a result, we found that a trapezoidal wing planform with the parameters shown in table 1 is equivalent to an actual butterfly's wing planform in terms of the aerodynamic performance factors such as lift force, thrust force, pitching moment, power expenditure, and power loading. In addition, we found that the resulting trapezoidal wing planform has almost the same flight behavior as the actual butterfly's wing planform. From these results, we can conclude that our hypothesis is valid, and we believe that these four parameters can be useful in designing butterfly-like MAVs.

\section{acknowledgments}

This work was supported by JSPS KAKENHI Grant Number JP16K18012. The authors would like to thank Mr Y. Yoshida for help with the simulations of the free flight in section 5.2.

\section{References}

Ancel, A. O., Eastwood, R., Vogt, D., Ithier, C., Smith, M., Wood, R. \& Kvač, M. (2017). Aerodynamic evaluation of wing shape and wing orientation in four butterfly species using numerical simulations and a low-speed wind tunnel, and its implications for the design of flying micro-robots, Interface Focus 7: 20160087 (13pp).

Barbarino, S., Bilgen, O., Ajaj, R. M., Friswell, M. I. \& Inman, D. J. (2011). A review of morphing aircraft, J. Intell. Mater. Syst. Struct. 22: 823-877.

Berwaerts, K., Matthysen, E. \& van Dyck, H. (2008). Take-off flight performance in the butterfly Pararge aegeria relative to sex and morphology: a quantitative genetic assesment, Evolution 62: 2525-2533.

Berwaerts, K., van Dyck, H. \& Aerts, P. (2002). Does flight morphology relate to flight performance? An experimental test with the butterfly Pararge aegeria, Funct. Ecol. 16: 484-491.

Betts, C. R. \& Wootton, R. J. (1988). Wing shape and flight behaviour in butterflies (Lepidoptera: Papilionoidea and Hesperioidea): a preliminary analysis, J. Exp. Biol. 138: 271-288.

Combes, S. A. \& Daniel, T. L. (2003). Into thin air: contributions of aerodynamic and inertial-elastic forces to wing bending in the hawkmoth Manduca sexta, J. Exp. Biol. 206: 2999-3006.

Dudley, R. (1990). Biomechanics of flight in neotropical butterflies: morphometrics and kinematics, J. Exp. Biol. 150: 37-53.

Dudley, R. (2002). The Biomechanics of Insect Flight: Form, Function, Evolution, Princeton University Press, New Jersey.

Engels, T., Kolomenskiy, D., Schneider, K. \& Sesterhenn, J. (2016). FluSI: a novel parallel simulation tool for flapping insect flight using a Fourier method with volume penalization, SIAM J. Sci. Comput. 38: S3-S24. 
Fei, Y.-H. J. \& Yang, J. T. (2015). Enhanced thrust and speed revealed in the forward flight of a butterfly with transient body translation, Phys. Rev. E 92: 033004 (10pp).

Hunt, J. C. R., Wray, A. A. \& Moin, P. (1988). Eddies, stream, and convergence zones in turbulent flow, Proc. of the Summer Program 1988, pp. 193-208.

Inamuro, T. (2012). Lattice Boltzmann methods for moving boundary flows, Fluid Dyn. Res. 44: 024001 (21pp).

Kingsolver, J. G. (1999). Experimental analyses of wing size, flight, and survival in the western white butterfly, Evolution 53: 1479-1490.

Shyy, W., Aono, H., Chimakurthi, S. K., Trizila, P., Kang, C. K., Cesnik, C. E. S. \& Liu, H. (2010). Recent progress in flapping wing aerodynamics and aeroelasticity, Progress in Aerospace Sciences 46: $284-327$.

Shyy, W., Lian, Y., Tang, J., Viieru, D. \& Liu, H. (2008). Aerodynamics of low Reynolds number flyers, Cambridge University Press, New York.

Srygley, R. B. (1999). Locomotor mimicry in Heliconius butterflies: contrast analyses of flight morphology and kinematics, Phil. Trans. R. Soc. Lond. B 354: 203-214.

Srygley, R. B. \& Thomas, A. L. R. (2002). Unconventional lift-generating mechanisms in free-flying butterflies, Nature 420: 660-664.

Sunada, S., Kawachi, K., Watanabe, I. \& Azuma, A. (1993). Performance of a butterfly in take-off flight, J. Exp. Biol. 183: 249-277.

Suzuki, K. \& Inamuro, T. (2011). Effect of internal mass in the simulation of a moving body by the immersed boundary method, Comput. Fluids 49: 173-187.

Suzuki, K., Minami, K. \& Inamuro, T. (2015). Lift and thrust generation by a butterfly-like flapping wing-body model: immersed boundary-lattice Boltzmann simulations, J. Fluid Mech. 767: 659695.

Suzuki, K. \& Yoshino, M. (2017). Aerodynamic comparison of a butterfly-like flapping wing-body model and a revolving-wing model, Fluid Dyn. Res. 49: 035512 (26pp).

Suzuki, K. \& Yoshino, M. (2018). Numerical simulations for aerodynamic performance of a butterflylike flapping wing-body model with various wing planforms, Commun. Comput. Phys. 23: 951979.

Taylor, G. K., Nudds, R. L. \& Thomas, A. L. R. (2003). Flying and swimming animals cruise at a Strouhal number tuned for high power efficiency, Nature 425: 707-711.

Triantafyllou, G. S., Triantafyllou, M. S. \& Grosenbaugh, M. A. (1993). Optimal thrust development in oscillating foils with application to fish propulsion, J. Fluids Struct. 7: 205-224.

Wang, Z. J. (2000). Vortex shedding and frequency selection in flapping flight, J. Fluid Mech. 410: 323341.

Warren, A. D., Davis, K. J., Grishin, N. V., Pelham, J. P. \& Stangeland, E. M. (accessed 2013-10-14). Interactive listing of american butterflies, 30-XII-12 p. http://www.butterfliesofamerica.com/.

Weis-Fogh, T. (1973). Quick estimates of flight fitness in hovering animals, including novel mechanisms for lift production, J. Exp. Biol. 59: 169-230.

Yokoyama, N., Senda, K., Iima, M. \& Hirai, N. (2013). Aerodynamic forces and vortical structures in flapping butterfly's forward flight, Phys. Fluids 25: 021902 (24pp).

Zhang, L., Hedrick, T. L. \& Mittal, R. (2013). Time-varying wing-twist improves aerodynamic efficiency of forward flight in butterflies, PLOS ONE 8: e53060 (10pp). 